\title{
Educación emocional en adultos y personas mayores
}

\author{
Belén López-Pérez, Irene Fernández-Pinto, \\ María Márquez-González
}

Facultad de Psicología, Universidad Autónoma de Madrid

\section{España}

Belén López Pérez. C/ Fuentespina 3, 5 C, 28031 Madrid. España E-mail: bln_lpz@,hotmail.com $\overline{\text { (C) Education \& Psychology } \mathrm{I}}+\mathrm{D}+\mathrm{i}$ and Editorial EOS (Spain) 


\section{Resumen}

Este artículo parte de la escasa investigación disponible hasta la fecha acerca del funcionamiento de las emociones en la edad adulta y la vejez y, particularmente, de los programas de intervención orientados a mejorar las competencias emocionales en estos grupos de edad. Para proponer un programa estructurado, se ha partido del modelo de inteligencia emocional propuesta por Salovey y Mayer (1990), introducido ciertas variaciones, y tomado aspectos de otros programas ya desarrollados. Además de los contenidos y objetivos específicos a trabajar - atención emocional, facilitación emocional, comprensión emocional, regulación emocional, autoestima y habilidades sociales -, se propone la metodología desde la que abordar estos objetivos y los instrumentos de evaluación que permitirán una valoración de la eficacia del programa al obtener información acerca del cambio (mejora) en las competencias emocionales al finalizar el mismo. Con todo ello, se pretende llamar la atención sobre los beneficios de llevar a cabo intervenciones en estos grupos de población que han sido menos estudiados hasta el momento y proporcionar una herramienta útil para llevarlas a cabo partiendo de los conocimientos empíricos disponibles.

Palabras Clave: educación emocional, adultos, vejez y programas psicoeducativos de intervención.

Recibido: 05/04/08 Aceptación Provisional: 01/05/08 Aceptación Definitiva: 16/07/08 


\begin{abstract}
This article starts with the scarce research results available on emotional functioning in early, middle and late adulthood, and in particular, the paucity of intervention programs which seek to improve emotional competencies in these age groups. Drawing on Salovey and Mayer's emotional intelligence theory (1990) and other previous applied work, an intervention program is proposed here. The following are specified: objectives, specific contents (attention, facilitation of emotions, understanding emotions, regulating emotions and social skills), methodology for addressing these objectives and assessment instruments for evaluating program effectiveness, through measuring the change in the emotional competencies at the end of the intervention. The main aims of this study are, on one hand, to highlight the importance of carrying out interventions aimed at enhancing emotional competence in adulthood, a stage of life which has received less attention than childhood, and, on the other hand, to provide a useful program for doing so, based on the available empirical knowledge.
\end{abstract}

Keywords: emotional education, adulthood, elderly, and intervention programs

Received: 05/04/08 Initial Acceptance: 01/05/08～Final Acceptance: 16/07/08 


\section{Educación emocional en adultos y personas mayores}

Desde hace algo más de una década, las emociones, los afectos, los estados de ánimo han empezado a ganar en importancia tanto en el pensamiento científico como en la sociedad en general. Así, tal y como señala Zaccagnini (2004), la sociedad actual ha concedido un nuevo papel a las emociones: han dejado de ser algo negativo, a reprimir, para convertirse en un elemento positivo que ayuda a actuar y tomar decisiones y, por tanto, a adaptarse a la vida cotidiana.

Durante la etapa adulta se producen múltiples cambios de distinta índole (a nivel biológico, cognitivo y social). Por todo ello, es muy importante que la persona tenga recursos para prevenir algunos de estos cambios y prepararse para afrontar o adaptarse a la mayoría de ellos. Si bien los cambios físicos y cognitivos son fácilmente identificables, no ocurre lo mismo con los cambios en el funcionamiento emocional (Lawton, 2001; Levenson, 2000). Diversos estudios han encontrado cambios en aspectos emocionales tales como, la optimización del afecto y la complejidad del mismo (Labouvie-Vief, Diel, Jain y Zhang, 2007), la intensidad emocional (Márquez-González, Izal, Montorio y Losada, en prensa) o el uso de diferentes estrategias de regulación emocional (Chapman y Hayslip, 2006; Márquez González et al., en prensa) entre otros. Una revisión de los estudios internacionales sobre las particularidades de la experiencia y regulación emocional en la etapa de la vejez puede encontrarse en Márquez, Izal, Montorio y Pérez-Rojo (2004).

La mayor parte de los trabajos de educación emocional y social han ido dirigidos a niños y jóvenes, debido a la importancia esencial de estas etapas evolutivas en la consolidación de las estructuras de funcionamiento psicosocial, así como en la prevención de problemas psicológicos futuros (Bellamy, Gore y Sturgis, 2005; Trianes, Cardelle-Elawar, Blanca y Muñoz, 2003). No obstante, las personas adultas y mayores también podrían beneficiarse ampliamente de estos programas, que suponen una oportunidad, bien para aprender competencias que nunca llegaron a adquirir por diferentes razones, o bien para potenciar o mejorar competencias adquiridas pero no desarrolladas plenamente. Según Grewall, Brackett y Salovey (2006), la inteligencia emocional está compuesta, más que por competencias de personalidad de carácter estable o fijo, por habilidades que pueden aprenderse a lo largo del ciclo vital a través del entrenamiento adecuado. En este sentido, una adecuada educación emocional es de gran importancia para lograr una adecuada 
socialización (Bisquerra, 2000), mejorar la calidad de vida y como factor protector ante problemas no sólo psicológicos sino también médicos que pueden aparecer a lo largo de la vida (Richman, Kubzansky, Maselko, Kawachi, Choo, Bauer, 2005). Se han desarrollado numerosas intervenciones en el ámbito escolar y las organizaciones dirigidas a incrementar la competencia emocional de los participantes (Havighur, Harley y Prior, 2004; Margot, Schick y Cierpka, 2003; Vadeboncoeur y Huguette; 2005). Asimismo, también se están llevando a cabo algunos programas de educación emocional orientados a mayores. Un buen ejemplo es el programa emociona't (Soldevilla, Ribes, Filella y Agulló, 2005), que en forma de taller trabaja distintas habilidades emocionales.

La educación emocional es importante tanto en la edad adulta como en la vejez, ya que ambos grupos muestran dificultades o déficits en alguno de los componentes de la inteligencia emocional. Así, Vasiliki y Louise (2008) han señalado que en la vejez aumentan las dificultades en el reconocimiento de emociones de signo negativo como la tristeza o el miedo. En este sentido, Thomas (2002) ha señalado que aunque aumenta la dificultad en el reconocimiento, por otro lado en la vejez disminuye la experimentación de la ira. Sin embargo, Matthias, Theodor y Louise (2007) han señalado que las personas de tercera edad muestran mejores estrategias de reparación emocional que los adultos. Por otro lado, Heckman y Blanchard-Fields (2008) han señalado que aunque las personas mayores lleven a cabo más estrategias de regulación, éstas son pasivas. Además, estos autores han señalado que en la vejez a diferencia de la edad adulta disminuye la habilidad para integrar cognición y emoción. Así, Labouvie-Vief, Dile, Jain y Zhang (2007) señalan que tanto la optimización como la complejidad afectiva tienden a disminuir a partir de los 60 y 45 años respectivamente.

Todos estos estudios llevan a plantear varias conclusiones: en primer lugar, en la edad adulta será necesario trabajar especialmente en la promoción de estrategias adecuadas de regulación emocional, especialmente en relación con las emociones de signo emocional negativo; en segundo lugar, en la vejez habrá que hacer énfasis en el componente de atención emocional para mejorar el reconocimiento emocional, en la facilitación emocional para trabajar la conexión de pensamientos y emociones y, finalmente, en la regulación emocional promoviendo el desarrollo de mecanismos más elaborados. 
Una de las definiciones de educación emocional más aceptadas es la de Bisquerra (2000). Para este autor, la educación emocional es "un proceso educativo, continuo y permanente, que pretende potenciar el desarrollo emocional como complemento indispensable del desarrollo cognitivo, constituyendo ambos los elementos esenciales del desarrollo de la personalidad integral. Para ello se propone el desarrollo de conocimientos y habilidades sobre emociones con objeto de capacitar al individuo para afrontar mejor los retos que se plantean en la vida cotidiana. Todo ello tiene como finalidad aumentar el bienestar personal y social" (p. 243).

Esta completa definición hace hincapié en una serie de aspectos muy importantes. Por una parte, entiende el desarrollo emocional como complementario del desarrollo cognitivo. En este sentido, se plantea que existe una relación bidireccional entre emoción y cognición (Salovey y Mayer, 1997) y, por ello, potenciando el desarrollo emocional del individuo también se está ejerciendo un efecto favorable sobre sus capacidades cognitivas. Por otra parte, Bisquerra (2000) entiende el desarrollo emocional como desarrollo de conocimientos y habilidades emocionales, lo cual tiene una aplicación práctica directa a la hora de desarrollar un programa de educación emocional: se debería promover un aprendizaje tanto conceptual como procedimental de las diferentes competencias que se desee enseñar. En cualquier caso, no debe perderse de vista que los programas de educación emocional tienen como objetivo último fomentar el bienestar personal y social del individuo y optimizar su calidad de vida.

A continuación se pasará a describir una propuesta teórica de un programa de educación emocional en adultos y mayores.

\section{Definición, marco teórico y objetivos}

Un programa de educación emocional debe basarse en el conocimiento y evidencia empírica disponible, siendo una herramienta al servicio del desarrollo de la inteligencia emocional de las personas. La inteligencia emocional es "la habilidad para percibir, generar y acceder a emociones que faciliten el pensamiento, para comprender el conocimiento emocional y para regular las emociones de manera efectiva permitiendo el crecimiento intelectual y emocional" (Mayer, Salovey y Caruso, 2004, p.15). Por tanto, se pueden 
identificar cuatro componentes básicos: atención, facilitación, comprensión y regulación emocional (Salovey y Mayer, 1997).

Se va a trabajar partiendo de una visión muy próxima a las desarrolladas por Extremera, Fernández-Berrocal y Ramos (2004) y Salovey y Mayer (1990/1997), ya que se trata de perspectivas teóricamente coherentes, con apoyo empírico sólido y cuya estructura parsimoniosa es susceptible de ser plasmada en un programa concreto y didáctico de educación emocional. Al mismo tiempo, un programa de educación emocional también debe fundamentarse en los amplios conocimientos acumulados por la ciencia psicológica en relación con las emociones, su naturaleza y su funcionamiento, sin dejar de lado la variabilidad interpersonal y la consiguiente necesidad de adaptarse a las necesidades particulares de cada grupo y de cada persona (Soldevila, 2003).

Las áreas propuestas y los objetivos para trabajar en un programa de educación emocional para adultos son:

- Adquirir un mejor conocimiento de las propias emociones, y en particular de las emociones derivadas de la adaptación al proceso de desarrollo a lo largo del ciclo vital: Conciencia o Atención Emocional.

- Conocer con mayor profundidad la relación entre las emociones y los pensamientos y otros procesos cognitivos: Facilitación Emocional

- Reconocer y comprender los estados emocionales propios y los de los demás y los procesos que los conforman: Comprensión Emocional

- Conocer y aplicar nuevas estrategias de regulación emocional, incluyendo el desarrollo de habilidades para generar emociones positivas y de habilidades para automotivarse

- Mejora de la autoestima

- Aprender habilidades sociales para mejorar la calidad de las relaciones interpersonales.

El programa de educación emocional busca, a través de estos objetivos, el cumplimiento de estos tres objetivos más generales:

- Potenciar la adaptación, la calidad de vida y el bienestar emocional y social de las personas.

- Favorecer las competencias cognitivas y emocionales para facilitar la adaptación y el afrontamiento de problemas.

- Prevenir el desarrollo de problemas emocionales (p.e. ansiedad, depresión, etc.) 
A continuación se desarrolla la estructura de un programa de educación emocional en adultos y mayores. Cada módulo está formado por una etapa educativa y una etapa de desarrollo de la competencia.

\section{Contenidos y Objetivos Específicos}

\section{Atención emocional}

La atención emocional consiste en prestar adecuada atención a los sentimientos propios y de los demás (ver Zaccagnini, 2004). Para que la atención sea algo saludable se debe trabajar para que la focalización atencional se produzca en un nivel intermedio, ya que tanto los bajos como los altos niveles atencionales dificultan e incluso impiden llevar a cabo cualquier tipo de actuación sobre las emociones (Zaccagnini, 2004).

El módulo de Atención incluye una fase educativa sobre el papel de las emociones en la vida, los tipos de emociones y el funcionamiento de éstas, y se hace especial énfasis en los cambios emocionales que se producen ligados al proceso de desarrollo. Así, los objetivos fundamentales de este módulo se pueden resumir en los siguientes:

- Conocer qué es y qué no es una emoción.

- Desarrollar un vocabulario emocional más preciso.

- Diferenciar entre el componente fisiológico, el cognitivo y el comportamental de la emoción.

- Saber aplicar esa diferenciación tanto a las emociones propias como a las de los demás.

\section{Facilitación emocional}

La facilitación emocional es entendida como la habilidad para asimilar la emoción en el pensamiento. Así, las emociones permiten priorizar el pensamiento y facilitan los juicios y la memoria (Salovey y Mayer, 1997). Este componente es especialmente relevante, ya que establece una bidireccionalidad entre la emoción y el pensamiento. De esta forma, a la vez que se trabaja la emoción se están desarrollando capacidades cognitivas. 
La fase educativa se centra en los siguientes aspectos: la influencia de la emoción en el pensamiento, la importancia de ser conscientes del efecto de las diferentes emociones sobre los propios pensamientos (p.e. atribuciones) y las estrategias para potenciar las emociones con efectos positivos y contrarrestar las emociones con efectos negativos. Los objetivos a alcanzar con este módulo se pueden resumir en los siguientes:

- Reflexionar sobre la influencia de las emociones sobre el pensamiento.

- Distinguir las emociones que potencian el pensamiento frente a las que no lo potencian e incluso lo obstaculizan.

- Saber aplicar estrategias para potenciar las emociones con efectos positivos y debilitar las que tienen efectos negativos.

\section{Comprensión emocional}

La comprensión emocional consiste en la capacidad para reconocer y entender las emociones propias y de los demás (Zaccagnini, 2004). A la hora de trabajar este componente hay que prestar atención a dos componentes diferentes: por un lado, la comprensión emocional intrapersonal y, por otro lado, la comprensión emocional interpersonal.

En primer lugar, se trabajará la faceta intrapersonal y se llevará a cabo una fase educativa centrada en la subjetividad de las emociones, en las fuentes posibles para identificar las emociones (aspectos verbales y no verbales), la importancia de una adecuada identificación y sus efectos, así como en los factores que ayudan a comprender qué es lo que se está sintiendo: identificación de los antecedentes, de los componentes y los consecuentes de esa emoción. Así, los objetivos más relevantes a trabajar son:

- Tomar conciencia de la subjetividad de las emociones.

- Identificar las emociones que uno mismo experimenta.

- Reconocer y discriminar entre distintas emociones, reconocer sus matices y ser conscientes de la existencia de emociones más ambiguas.

- Saber identificar las causas, componentes y consecuentes de una emoción propia. 
En segundo lugar, se trabajará el componente interpersonal de la comprensión emocional, a saber: la empatía. Aunque la empatía es un constructo multidimensional que engloba tanto un aspecto cognitivo como un aspecto afectivo (Davis, 1980), los programas de educación emocional se han centrado fundamentalmente en el desarrollo de la vertiente cognitiva de la empatía, más susceptible de modificación (Pérez, Martínez, Luque y Redondo, 2005). Los componentes a trabajar, siguiendo la estructura de López-Pérez, Fernández-Pinto y Abad (2008), son la adopción de perspectivas o capacidad para ponerse en el lugar de otra persona y la comprensión emocional o capacidad para identificar y comprender emociones en los demás.

Así, en la fase educativa se explicaría la diferencia entre adoptar el punto de vista del otro y comprender las emociones del otro, se enfatizaría otra vez sobre la subjetividad de las emociones y se explicarían estrategias para facilitar la adopción de perspectivas (p.ej. imaginarse a uno mismo en esa situación) y la comprensión emocional (p.ej. identificación de los posibles antecedentes de la emoción). Los objetivos a alcanzar en este módulo son:

- Saber identificar diferentes emociones en los otros

- Saber identificar las posibles causas y consecuencias de las emociones en los otros

- Saber expresar cómo se puede sentir una persona ante una situación determinada

- Saber expresar cómo se sentiría él mismo en esa situación

Trabajar este componente es, junto con la regulación emocional, uno de los aspectos más relevantes del programa. La empatía juega un papel muy relevante en las relaciones interpersonales (Davis y Oathout, 1987), ya que promueve la conducta prosocial y de afiliación (Mehrabian y Epstein, 1972), entre otras. Sin embargo, al igual que en el componente de atención, el objetivo del programa sería intentar promover unos niveles intermedios de empatía, ya que parecen ser los más óptimos (Eisenberg, Fabes, Nyman, Bernzweig y Pinuelas, 1994).

\section{Regulación emocional}

La regulación emocional también recibe el nombre de reparación de los estados de ánimo y ha sido definida como la habilidad de las personas para influir en qué emociones tienen, cuándo las tienen y cómo las experimentan y las expresan (Eisenberg, Fabes, Guthrie y Reiser, 2000), o también como cualquier intento que hacen las personas para modificar en alguna medida la ocurrencia, intensidad o duración de un estado emocional (positivo o 
negativo), bien alterando alguno de los factores que anteceden a la emoción (p.ej., situaciones antecedentes), bien modificando algún aspecto de la emoción en sí misma (p.ej., supresión de la respuesta emocional) (Gross, 1998). Si bien se ha señalado que es muy difícil especificar de forma clara qué estrategias específicas de regulación emocional son mejores o preferibles a otras, algunos investigadores han encontrado que existen diferencias claras entre algunas estrategias de regulación en cuanto a sus correlatos de salud y bienestar tanto psicológico como físico (Gross, 1998). Así, en la vertiente negativa, el estilo represivo de afrontamiento o la inhibición de la experiencia emocional subjetiva (supresión emocional) presenta efectos negativos sobre la salud física (Cano-Vindel, Sirgo y Díaz-Ovejero, 1999) y la rumiación ante estados de tristeza parece perpetuar el estado depresivo (Lyubomirsky y Nolen-Hoeksema, 1993). En cuanto a las estrategias regulatorias con mayor potencial adaptativo, se han considerado la revaluación cognitiva y las técnicas de pensamiento racional (ver Vázquez, 2003), las técnicas de manejo del estrés (p.ej., inoculación de estrés; Meichenbaum, 1987), la relajación y el ejercicio físico (Thayer, Newman, y McClain, 1994) y la expresión emocional a través de la escritura (Pennebaker, 1997).

La incorporación de este módulo en un programa de desarrollo emocional responde al objetivo de transmitir a las personas la noción de que tienen capacidad de influencia sobre sus propias emociones y que puede aprender distintas estrategias y desarrollar recursos para modificar éstas. También se trata en este módulo de enseñar a la persona a aumentar sus emociones positivas. En la fase educativa se explicaría en qué consiste la regulación emocional, se, explicarían qué técnicas se pueden emplear y qué utilidad tiene cada técnica en función de cada situación. Los objetivos a trabajar en este módulo son:

- Que la persona observe ejemplos concretos, basados en su propia experiencia, de cómo tiene capacidad de influir sobre sus estados emocionales.

- Que la persona aprenda diferentes estrategias concretas para regular sus emociones.

- Que la persona ponga en práctica esas estrategias para llevar a cabo la regulación emocional.

\section{Autoestima}

La autoestima consiste en la actitud favorable o desfavorable que una persona tiene sobre sí misma (Blascovich y Tomaka, 1991). Por ello, la autoestima es algo fundamental a trabajar en un programa de educación emocional. La fase educativa del programa irá dirigida a trabajar sobre el autoconcepto de cada persona, sus competencias personales observables y 
las reconocidas socialmente, sus logros personales y, en relación con éstos, sus roles, metas y objetivos. El trabajo de estos aspectos resulta esencial en cualquier edad de cara a potenciar el reajuste adaptativo del autoconcepto.

Así, teniendo en cuenta el programa Emociona't (Soldevila et al., 2005) se proponen los siguientes objetivos:

- Reconocer los indicadores de una autoestima equilibrada.

- Tomar conciencia del nivel de autoestima propio y de los demás.

- Desarrollar expectativas positivas sobre uno mismo.

- Conocer qué aspectos valoran los demás de uno mismo.

- Aceptar la imagen que los demás tienen de uno mismo.

- Reconocer las propias habilidades y aptitudes.

- Aumentar las competencias personales a nivel visible.

- Utilizar estrategias para que los demás valoren las propias habilidades y se incremente el valor social propio.

En este caso, al igual que en otros de los componentes a trabajar, es necesario realizar, en primer lugar, intervenciones a nivel cognitivo (p.ej. en el estilo atribucional) para que posteriormente se puedan apreciar cambios a nivel emocional.

\section{Habilidades sociales}

Las relaciones interpersonales son fundamentales para el bienestar. En cualquier edad, la existencia de una red de apoyo de calidad en torno a la persona es un buen predictor de su salud física y mental (Hale, Hannum y Espelage, 2005). Por ello, tener habilidades para adquirir, mantener y fomentar las relaciones interpersonales, como un modo de ampliar cuantitativa y cualitativamente la red de apoyo, debe ser un objetivo fundamental en cualquier programa destinado a mejorar el bienestar de las personas.

En la fase educativa se debe explicar qué habilidades conforman las denominadas habilidades sociales, la influencia de las emociones sobre las habilidades sociales y la importancia de unas buenas habilidades sociales. Este módulo se estructura en torno a los siguientes objetivos: 
- Conocer los distintos estilos de comunicación interpersonal (asertivo, agresivo y pasivo).

- Aprender a reconocer cuál es el estilo predominante en uno mismo.

- Reconocer el papel que juegan las emociones en las relaciones interpersonales.

- Conocer y practicar la escucha activa.

- Aprender a pedir y conseguir ayuda de los demás.

\section{Contexto, principios de intervención y temporalización}

Este programa se puede desarrollar en diferentes contextos, ya que son múltiples los ámbitos en los que interesa desarrollar las competencias emocionales en los adultos y las personas mayores. En particular, en el caso de los adultos, el ámbito de aplicación en el que puede tener mayor cabida este tipo de intervención es el organizacional, en el que poco a poco se están introduciendo más programas destinados a mejorar las competencias de los empleados con el objetivo de mejorar la productividad y las relaciones interpersonales. En este sentido, la educación emocional es fundamental de cara a la satisfacción personal de los empleados así como al fomento del desarrollo de las relaciones sociales en el seno de la organización. No obstante, también existen otros ámbitos de aplicación, como por ejemplo las sesiones de terapia grupales en el contexto clínico. En la población de personas mayores, también es creciente la implementación de talleres de diversa índole en centros municipales, centros de día, etc. En este sentido, este programa puede desarrollarse siguiendo este mismo formato de taller ya que, como se comentó anteriormente, la educación emocional tiene una gran importancia en esta etapa de la vida.

El programa está basado en dos tipos de principios psicológicos. Por una parte, los principios constructivistas, ya que en todas las sesiones se procura partir del conocimiento previo de los asistentes al programa y resumir la información al final de cada sesión. Por otra parte, se trabajan técnicas de intervención típicamente cognitivo-conductuales en distintos momentos del programa y, particularmente, en el módulo de regulación emocional.

El programa propuesto se organiza en torno a 6 módulos, por lo que se propone una intervención de unas 18-20 sesiones. Cada sesión tendrá una duración aproximada de unos 90 
minutos. La estructuración de cada sesión sería: explicar los objetivos de la sesión (5 min.), fase educativa (20 min.), entrenamiento (60 min.) y resumen de las ideas claves (5 min.).

\section{Sistema de evaluación}

Se llevarán a cabo tres tipos de evaluación. La evaluación inicial, la evaluación del proceso y la evaluación final.

\section{La evaluación inicial}

$\mathrm{Su}$ objetivo es establecer la línea base de la que parten los participantes en las diferentes variables a intervenir, antes de llevar a cabo la intervención. En ella se podrán emplear diferentes instrumentos. Por una parte, es posible aplicar medidas globales de inteligencia emocional. También se pueden evaluar por separado cada uno de los diversos componentes. Para ello, se puede utilizar el TMMS-24 (Trait Meta Mood Scale-24; Fernández-Berrocal, Extremera y Ramos, 2004) para medir la inteligencia emocional en relación a los componentes de atención, claridad (comprensión emocional) y regulación emocional. Para la evaluación de la empatía cognitiva es posible utilizar el cuestionario EM (Hogan Empathy Scale; Hogan, 1969) o las escalas cognitivas del IRI (Índice de Reactividad Interpersonal; Davis, 1980) o del TECA (Test de Empatía Cognitiva y Afectiva; LópezPérez, Fernández-Pinto y Abad, 2008). La autoestima se puede evaluar a través del GSE (Global Self-Esteem Scale; Rosenberg, 1979). Para las habilidades sociales una posible medida es realizar el rol playing estructurado propuesto por Ikebuchi, Miyauchi, Anzai, Kumagai, Hata, Honjoh, Amigasa y Maeda (1994). Finalmente, puesto que la finalidad de la educación emocional es mejorar el bienestar de la persona, se puede evaluar el éxito del programa utilizando alguna medida de bienestar como puede ser la de Ryff (1989). Sin embargo, todas estas medidas pueden no ser sensibles al cambio experimentado por el programa ya que miden rasgos o tendencias y, por lo tanto, sería más adecuado emplear medidas de habilidad, como por ejemplo el MSCEIT v.2.0. (Mayer, Salovey y Caruso, 2002; adaptación al castellano por Extremera y Fernández-Berrocal, 2002), que permitiría evaluar los niveles de Percepción, Facilitación, Comprensión y Manejo emocional. 


\section{La evaluación del proceso}

Permite analizar si el programa se está aplicando según lo previsto y si se están alcanzando los objetivos intermedios, establecidos como primer paso para alcanzar los objetivos finales. Para ello, se puede realizar una pequeña evaluación al final de las sesiones o cada dos sesiones en la que se pregunte a los participantes sobre la utilidad de la sesión, si cambiarían algo o no, su opinión sobre el ejercicio del terapeuta, etc. De la misma forma, se puede elaborar un pequeño registro con los objetivos intermedios y a través de la observación ir registrando cuáles han sido alcanzados y cuáles no por cada participante y cuáles han sido las dificultades encontradas. También se puede evaluar a través de tutorías semanales o quincenales, para llevar a cabo un seguimiento más exhaustivo.

\section{La evaluación final}

Se volverán a tomar medidas de las mismas variables evaluadas inicialmente, a través de los mismos instrumentos ya mencionados. Esta medida permitirá ver en qué medida se han producido cambios gracias a la intervención.

\section{Discusión}

Al inicio de este trabajo, se resaltaba la importancia de las emociones y sus posibles correlatos sobre la salud y el bienestar de las personas de cualquier edad, destacando los beneficios que presenta, de cara al bienestar y la adaptación personal y social, el que la persona cuente con unos niveles adecuados de inteligencia emocional que le permitan conocer, comprender y actuar de manera eficaz sobre las emociones propias y de los demás. Igualmente, se ha hecho explícita la escasez de programas de intervención orientados hacia este objetivo, escasez especialmente patente en la etapa adulta de la vida, ya que la mayor parte de los programas de educación emocional se han desarrollado en el ámbito escolar. Sin embargo, las modernas concepciones de la inteligencia emocional comentadas en este trabajo sustentan la validez y conveniencia del entrenamiento en este conjunto de habilidades en cualquier etapa del ciclo vital, ya que éstas son concebidas como destrezas que pueden ser aprendidas, y no como competencias fijas ancladas en los engranajes de la personalidad de cada individuo. 
En base a los modelos y consideraciones propuestas en los sólidos trabajos realizados por Mayer y Salovey (1997) y Bisquerra (2000), se ha presentado en este trabajo un esquema de los contenidos y procedimientos básicos a incluir en un programa de educación emocional dirigido a población adulta y que, por tanto, ha sido diseñado teniendo en cuenta las características y necesidades de esta población. Esta es la principal aportación y el objetivo fundamental de este trabajo: proporcionar un esquema de trabajo útil para este grupo de población que, hasta la fecha, ha recibido menor atención en lo referente a la educación emocional y que puede beneficiarse ampliamente de ella. Lo que aquí se propone no son técnicas novedosas sino una estructura de programa basada en el paradigma de estudio de la inteligencia emocional, los conocimientos disponibles hasta la fecha y en otros programas ya desarrollados previamente con población adulta. Intencionadamente, el nivel de especificación de los procedimientos concretos a seguir no es elevado, ya que el trabajo tiene como objetivo ofrecer un guión-esquema del programa, que habrá de ser desarrollado y concretado en cada caso teniendo en cuenta las necesidades específicas de la población a la que vaya dirigido.

Uno de los objetivos fundamentales en el desarrollo de este programa ha sido el de ajustarse en la mayor medida posible al esquema de la inteligencia emocional planteado por Salovey y Mayer. De este modo, se pretende que cada uno de los módulos que se incluyen en este programa trabaje una competencia específica y claramente definida. Por ello, una de las novedades de este programa es el desarrollo de la facilitación emocional, que apenas había sido trabajado en otros programas y es posiblemente la faceta menos difundida de la estructura propuesta por Salovey y Mayer. Asimismo, se ha pretendido hacer una clara distinción entre los aspectos interpersonales y los intrapersonales, especialmente en el módulo de comprensión emocional. La comprensión emocional en muchos casos y de forma específica dentro del paradigma de la inteligencia emocional se ha restringido al ámbito de la comprensión intrapersonal, dejando de lado la comprensión interpersonal, que es uno de los componentes fundamentales de la empatía. La empatía es un aspecto fundamental a desarrollar en cualquier etapa del ciclo vital, puesto que fomenta las relaciones interpersonales. Sin embargo, en la última etapa de la vida las relaciones sociales adquieren una importancia aún mayor si cabe, por la tendencia de las mismas a declinar (muerte de amigos, cónyuge, etc.). 
Un aspecto destacable del programa aquí expuesto es la operativización de los procedimientos de evaluación. Se propone una evaluación anterior a la puesta en marcha del programa y una revaluación posterior. De este modo, se cumple una doble finalidad: por una parte es posible detectar las necesidades de las personas que integran los grupos con los que se va a trabajar. En segundo lugar, la evaluación posterior al programa permitirá conocef la evolución de las personas a las que se ha aplicado el programa y la posible existencia de algunas áreas que no se hayan desarrollado suficientemente, pero fundamentalmente permitirá poner a prueba la eficacia del programa y de cada uno de sus módulos y actividades,

Aún queda mucho por desarrollar en el campo de la educación emocional, pero, para que se produzca este desarrollo, una de las bases fundamentales es la concienciación acerca de la importancia de las emociones en todas las edades, incluidas la edad adulta y la vejez, para mejorar el bienestar de las personas. Para ello, es necesario generar instrumentos que, además de basarse en los conocimientos empíricos disponibles, se ajusten a las necesidades prácticas de las personas que los van a llevar a cabo así como a las características de la población a la que se dirigen. Este es el objetivo que se ha tratado de lograr aquí proponiendo técnicas sencillas y específicas, pero aún queda mucho por hacer y para ello es necesario realizar un mayor esfuerzo investigador para conocer las características específicas de las personas adultas y mayores en cuanto a su experiencia y regulación emocional. 


\section{Referencias}

Bellamy, A., Gore, D. y Sturgis, J. (2005) Una exploración de la relevancia de la Inteligencia emocional en alumnos con alta capacidad. Electronic Journal of Research in Educational Psychology, 3(2), 53-78.

Bisquerra, R. (2000). Educación emocional y bienestar. Barcelona: Praxis S. A.

Blascovich, J. y Tomaka, J. (1991). Measures of self-esteem. En Robinson, J. P., Shaver, P. R. y Wrightsman, L. S. Measures of personality and social psychological attitures. Measures of social psychological attitudes, Vol. 1. (pp. 115-160). San Diego, CA, US: Academic Press.

Cano-Vindel, A., Sirgo, A. y Díaz Ovejero, M. B. (1999). Control, defensa y expresión de emociones: relaciones con salud y enfermedad. En E. G. Fernández-Abascal y F. Palmero (Eds.), Emociones y Salud (pp. 69-90). Madrid: Ariel.

Davis, M. H. (1980). A multidimensional approach to individual differences in empathy. Catalog of Selected Documents in Psychology, 10, 85, (pp.1-17)

Davis, M. H. y Oathout, H. A. (1987). Maintenance of satisfaction in romantic relationships: Empathy and relational competence. Journal of Personality and Social Psychology 53 (2), $397-410$.

Eisenberg, N., Fabes, R. A., Nyman, M. y Bernzweig, J. y Pinuelas, A. (1994). The relations of emotionality and regulation to children's anger-related reactions. Child Development, 65(1), 109-128.

Eisenberg, N., Fabes, R. A., Guthrie, I. K. y Reiser, M. (2000). Dispositional emotionality and regulation: their role in predicting quality of social functioning. Journal Personality and Social Psychology, 78 (1), 136-157.

Extremera, N. y Fernández-Berrocal, P. (2002). Cuestionario MSCEIT (Versión española 2.0) de Mayer, Salovey y Caruso. Toronto, Canada: Multi-Health Systems Publishers.

Fernández-Berrocal, P., Extremera, N. y Ramos, N. (2004). Validity and reliability of the Spanish modified version of the Trait Meta-Mood Scale. Psychological Reports, 94, 751-755

Fernández-Berrocal, P., Extremera, N., y Ramos, N. (2004). Validity and reliability of the Spanish modified version of the Trait Meta-mood Scale. Psychological Reports, 94, 751-755. 
Grewal, D. D., Brackett, M., y Salovey, P. (2006). Emotional intelligence and the selfregulation of affect. En D.K. Snyder, J.A. Simpson, y J.N. Hughes (Eds.), Emotion regulation in couples and families (pp. 37-55). Washington, DC: American Psychological Association.

Gross, J. J. (1998). Antecedent and response-focused emotion regulation: Divergent consequences for experience, expression, and physiology. Journal of Personality and Social Psychology, 74, 224-237.

Hale, C. J., Hannum, J. W. y Espelage, D. L. (2005). Social support and physical health: the importance of belonging. Journal of American College Health 53(6), 276284.

Havighurst, S. S.; Harley, A.; Prior, M. (2004). Building Preschool Children's Emotional Competence: A Parenting Program. Early Education and Development. Special Issue: Prevention Interventions with Young Children, 15(4), 423-447

Heckman, A. y Blanchard-Fields, F. (2008) Emotion Regulation in Interpersonal Problems: The Role of Cognitive-Emotional Complexity, Emotion Regulation Goals, and Expressivity. Psychology and Aging, 23 (1), 39-51.

Hogan, R. (1969). Development of an Empathy Scale. Counsulting and Clinical Psychology, 33, 307-316.

Ikebuchi, E., Miyauchi, M., Anzai, N., Kumagai, N., Hata, A., Honjoh, I., Amigasa, T. y Maeda, K. (1994). Assessment of social disability of patients patients suffering from chronic mental illness with the role play test. Seishin Shinkeigaku Zasshi, $96(3), 157-173$.

Labouvie-Vief, G., Diel, M., Jain, E. y Zhang, F. (2007). Six-Year Change in Affect Optimization and Affect Complexity Across the Adult Life Span: A Further Examination. Psychology and Aging, 22 (4), 738-751.

Lawton, M. P. (2001). Emotion in later life. Current Directions in Psychological Sciences 10(4), 120-123.

Levenson, R. W. (2000). Expressive, physiological, and subjective changes in emotion across adulthood. En Qualls, S. H. y Abeles, N. Psychology and the aging revolution: How we adapt to longer life. (pp. 123 - 140). Washington, DC, US: American Psychological Association. 
López-Pérez, B., Fernández-Pinto, I. y Abad, F. J. (2008). TECA. Test de Empatía Cognitiva y Afectiva. Madrid: TEA Ediciones.

Lyubomirsky, S. y Nolen-Hoeksema, S. (1993). Self-perpetuating properties of dysphoric rumination. Journal of Personality and Social Psychology, 65, 339349.

Márquez-González, M., Izal, M., Montorio, I. y Losada, A. (en prensa). Experiencia y regulación emocional a lo largo de la etapa adulta del ciclo vital: análisis comparativo en tres grupos de edad. Psicothema.

Márquez, M., Izal, M., Montorio, I., y Pérez Rojo, G. (2004). Emoción en la vejez: una revisión de la influencia de los factores emocionales sobre la calidad de la vida de las personas mayores. Revista Española de Geriatría y Gerontología, 39(3), 44-51.

Matthias, K., Theodor, J. y Louise, P.H. (2007) Emotional development across adulthood: Differential age-related emotional reactivity and emotion regulation in a negative mood induction procedure. International Journal of Aging \& Human Development, 64 (3), 217-244.

Mayer, J. D. y Salovey, P. (1997). What is Emotional Intelligence?. En Salovey, P. y Sluyter, D. J. Emotional Development and emotional intelligence: Educational implications (pp. 3-34). New York, NY, US: Basic Books.

Mayer, J. D., Salovey, P., y Caruso, D. R. (2002). Mayer-Salovey-Caruso Emotional Intelligence Test (MSCEIT) item booklet, Version 2.0. Toronto, Ontario, Canada: MHS Publishers.

Mayer, J. D., Salovey, P. y Caruso, D. R. (2004). Emotional intelligence: theory, findings and implications. Psychological Inquiry, 15, 197-215.

Mehrabian, A. y Epstein, N. (1972). A measure of Emotional Empathy. Journal of Personality, 40, 525-543.

Meichenbaum, D. (1987). Manual de inoculación del estrés. Barcelona: Martínez Roca.

Pennebaker, J. W. (1997). Opening Up. The Healing Power of Expressing Emotions. The Guilford Press. New York. London.

Pérez, M., Martínez, M., Luque, E. y Redondo, S. (2005). Agresores sexuales: perfiles de criminales y riesgo de reincidencia. Manuscrito no publicado. 
Richman, L. S., Kubzansky, L., Maselko, J., Kawachi, I., Choo, P. y Bauer, M. (2005). Positive Emotion and Health: Going Beyond the Negative. Health Psychology 24(4), 422 - 429.

Rosenberg, M. (1979). Conceiving the self. New York: Basic Books.

Ryff, C. D. (1989). Happiness is everything, or is it? Explorations on the meaning of psychological well-being. Journal of Personality and Social Psychology, 57(6), 1069-1081.

Salovey, P. y Mayer, J. D. (1990). Emotional intelligence. Imagination, Cognition and Personality, 9, 185-211.

Schick, A. y Cierpka, M. (2003) Evaluation of a curriculum to enhace social-emotional competente and prevent agresión in elementary schools. Kindheit und Entwicklung. Special Issue: Prävention von Verhaltensstörungen, 12 (2), 100110.

Soldevila, A. (2003). Los centros de día para personas mayores. Lleida, Publicaciones de la Universidad de Lleida.

Soldevila, A., Ribes, R., Filella, G. y Agulló, M. J. (2005). Objetivos y contenidos de un programa de educación emocional para personas mayores. Emociona’t. Revista Iberoamericana de Educación.

Thayer, R. E., Newman, J. R., y McClain, T. M. (1994). Self-regulation of mood: Strategies for changing a bad mood, raising energy, and reducing tension. Journal of Personality and Social Psychology, 67, 910-925.

Thomas, S.P. (2002) Age differences in anger frequency, intensity, and expression. Journal of the American Psychiatric Nurses Association, 8(2), 44-50.

Trianes, M. V., Cardelle-Elawar, M., Blanca, M. J y Muñoz, A. M. (2003). Contexto social, género y competencia social autoevaluada en alumnos andaluces de 11-12 años. Electronic Journal of Research in Educational Psychology, 1 (2), 37-56.

Vasiliki, O. y Louise, H. P. (2008) Effects of age and emotional intensity on the recognition of facial emotion. Experimental Aging Research, 34(1), 63-79

Vázquez, C. (2003). Procedimientos de intervención cognitiva. En Vázquez, C. (2003) Técnicas cognitivas de intervención clínica (pp. 51-88). Madrid: Síntesis. 
López-Pérez B. et al.

Zaccagnini, J. L. (2004) Qué es inteligencia emocional. Editorial Biblioteca Nueva: Madrid. 